\title{
SACRÉ ET TRANSCENDENCE: DisCOURS SPIRITUEL ET PHILOSOPHIQUE
}

\author{
[SACRED AND TRANSCENDENCE: PHILOSOPHICAL AND SPIRIT DISCURS]
}

\author{
Salim Mokaddem * \\ Université de Montpellier/Lirdef-Faculté d'éducation, France
}

\begin{abstract}
RÉSUMÉ: La philosophie est historiquement une pratique et une activité théorique autant que théorétique. Cependant, dans son histoire, elle a été contrainte, pour des raisons internes et externes à son histoire et à sa forme disciplinaire, autant qu'à son régime épistémologique, de se distinguer à la fois de la théologie et de la mystique ou de la spiritualité. Pourtant, elle se définissait dans sa généalogie comme une métaphysique ou une science de l'être et de la vérité. Comment s'articulent donc la transcendance et le sacré dans la praxis philosophique ? Quel rôle joue le rapport à soi et au dire vrai dans l'activité philosophique? Quelle place constitue l'histoire de la vérité et l'ontologie de soi dans la production des vérités en philosophie ? Et, enfin, que faut-il qu'engage de soi le sujet de l'action philosophique pour qu'il soit sans cesse confronté à inscrire son être dans une pratique de vérité et de savoir, et, en même temps, s'incarner existentiellement dans une logique où la liberté et la connaissance engagent des formes de vie spécifiques et singulières, propres à la vie philosophique définie comme rapport à soi, à l'autre, au monde?
\end{abstract}

Mots Cles: Discours spirituel; philosophie; opinion; vérité; transcendance; sacré; logos; éthique et métaphysique

\section{INTRODUCTION ET PROBLÉMATISATION}

$\mathrm{N}$ otre époque nihiliste et postmoderne refuse la question de la transcendance, et pour tout dire, la question du sens (histoire, sujet, existence) car elle se veut critique, et ancrée dans une position nihiliste, celle de la déconstruction (Derrida, 1967 ) et surtout, structurée, dans ses logiques de détermination catégoriale, par des

* Professeur de philosophie dans l'Université de Montpellier / Lirdef-Faculté d'éducation. Email: salim.mokaddem@gmail.com 
entrées conceptuelles matérialistes, au sens dialectique de la praxis et de l'humanisme feuerbachien. Mais, elle va plus loin encore que Feuerbach et même Marx, en s'arcboutant sur la thèse nietzschéenne de la « mort de Dieu » développée dans Ainsi parlait Zarathoustra, et du post-humanisme radical développé aujourd'hui par les sciences médicales et biologiques alliées aux technologies du numérique. Dans cette épistémè nihiliste, nulle essence, nulle réalité n'est situable hors espace-temps psychique et historique ; on peut faire remonter cette critique rigoureuse de la métaphysique à Kant qui territorialisa l'extension de la raison pure dans le champ spéculatif, pour éviter les errances et les illusions dogmatiques de la Schärmerei, qui est un enthousiasme délirant, en voulant appliquer l'usage de la raison théorique et pratique uniquement dans le champ des phénomènes. Le criticisme fait en effet disparaître le monde des noumènes dans une sphère pratique car inatteignable par la connaissance par concept. La raison doit donc alors se pencher désormais dans le monde sensible des phénomènes, situés dans l'espace et le temps, fussent-ils transcendantaux, mesurables et quantifiables par les catégories de l'entendement, et ainsi, la raison devra réfléchir sur les déterminations des choses, sur la constitution des objets de savoir, et sur les conditions de la production des connaissances scientifiques, des objets de la science et sur les fondements subjectifs des vérités critiques. Le transcendantal remplace le transcendant. Ce dernier relèvera de jugements relevant de l'action morale et du jugement réfléchissant. Kant ouvra la porte à l'infinitude de l'action en rendant le sujet à sa finitude définitive.

Pourquoi distinguer les deux champs, transcendance et sacré, philosophie et théologie, dans notre approche de la rationalité occidentale ? Pourquoi opposer discours spirituel et discours philosophique ? Cela a-t-il encore un sens aujourd'hui de déterminer deux domaines, deux régimes de discours et de vérité, de manière aussi formelle et binaire?

D'abord, explicitons, même brièvement, les notions en jeu, afin de bien comprendre les enjeux problématiques de cette question comparative :

- le sacré renvoie au profane : il peut donc y avoir du transcendant non sacré, c'est-à-dire, agissant dans nos vies quotidiennes, et dans notre présent le plus immédiat, sans ritualisations ou liturgies spécifiques. Donnons quelques exemples : les droits de l'enfant, ou la laïcité en France, ou les valeurs organiques d'une constitution juridique et extrajuridique d'un Etat ; de même, il existe du sacré sans transcendance. Exemples : les liturgies de respect des logiques ou des ordres hiérarchiques dans les institutions, le respect irréfléchi dans l'application des interdits moraux, ou la prescription et le suivi des tabous dans les us et coutumes des habitudes sociétales.

- le transcendant renvoie à un plan hors immanence, hors nature, monde, hors être (Dieu, l'Un de Plotin ou l'Idée du Bien chez Platon) et appelle donc à un dépassement sublime (sub-limes) des limites du Monde, des frontières de l'expérience, de la perception, voir de l'intuition (sensibleintelligible, corps-âme, nature-surnature, Cosmos-Theos, etc.) et à un audelà du sensible et de la nomination d'un élément du monde pour le désigner.

Il faut donc nous interroger sur les distinctions entre le spirituel (qui peut relever de la mystique ou de la spéculation métaphysique) et le philosophique de ce fait : car les distinctions et les différences ne sont pas uniquement entre l'ici-bas et l'au-delà, ou la transcendance et l'immanence. En effet, ce qui fut mystique dans l'Antiquité, et qui 
garde jusqu'à Hegel et dans les expressions diverses du romantisme allemand, dans son idéalisme spéculatif, cette part de transcendance, ne fut pas coupé de la science ou du monde des connaissances rationnelles. Qu'on pense à la gnose, ou à l'alchimie dont Koyré a étudié l'histoire afin de montrer que leurs concepts et leur approche épistémologique ont eu des rôles fondamentaux dans la constitution des sciences positives de la nature. La coupure apparaît vraiment de façon obvie avec Descartes et Leibniz qui scindèrent le champ du savoir en savoir physico-mathématique d'une part, et savoir théologico-philosophique, d'autre part, et qui pensèrent le destin de l'individu, du sujet, de la personne, dans un cadre téléologique et temporel à la fois de façon inédite et moderne, en ancrant le sujet dans une posture où il se dédouble en sujet de la science et en sujet métaphysique.

On peut trouver cette position affirmée de façon plus que thématisée dans le Traité Théologico-Politique de Spinoza qui reprend la critique cartésienne en l'appliquant aux textes religieux, en s'appuyant sur Averroès, notamment le Discours décisif, via Maïmonide et son Guide des égarés, et sa théorie des facultés (imagination, entendement, raison) et en développant de manière critique une herméneutique qui en grande partie, est rendue possible par la modernité protestante et sécularisatrice. En ce sens, la laïcité n'est pas contraire à la pratique religieuse, ni même à une certaine spiritualité, comme on le voit dans l'amour intellectuel de Dieu comme but de la philosophie selon Spinoza (Ethique, 1677). Il s'agit simplement, dans un processus de tolérance politique et juridique, de séparer exercice du pouvoir, visant au bien commun, de la foi, visant le salut individuel et relevant d'une rationalité qui échappe alors à la saisie par voie démonstrative de la vérité ou à la véracité avérée de tel ou tel culte.

Kant, dans les trois Critiques ira encore plus loin en faisant de la métaphysique une science impossible et de la théologie une illusion de la raison pure en ce sens qu'il nous est impossible de faire l'expérience de la chose en soi, du noumène, donc de Dieu, de l'âme, du Monde, autrement que sous la forme de la pratique et de la croyance en un Idéal transcendantal régulant nos actes moraux pour leur donner dignité, respect et sens, via la pratique du Bien dans l'impératif catégorique.

La question de la liberté de penser et celle de l'existence de la transcendance sont liées à la possibilité pour l'être humain de vivre le sublime et l'acte moral dans une vie pensée et pratiquée sous le chef du bien moral (Kant, 1787).

Légitimement, on peut pourtant s'interroger sur la possibilité de vivre une expérience de la transcendance dans un monde sécularisé et profane, d'une part, et marquer une différence ontologique (ou une différence qui ne soit pas ontothéologique) entre ce qui relève du spirituel et du théologique, d'autre part. Le travail précurseur de Guy Lardreau (Discours philosophique et discours spirituel) nous semble intéressant à reprendre à l'aune des thèses philosophiques sur la métahistoire développées par Henry Corbin (Temple et Contemplation).

Rappelons en la trame : il s'agit de montrer que ce qui différencie philosophie et spiritualité ne sont pas les objets des discours mais les formes, les pratiques, les modes existentiels de mises en action des vérités des disciplines. Il s'agit dans le fond de l'ontologie du sujet : les figures de la subjectivité sont des modalités métaphysiques d'être au monde, à soi, à l'autre, dans les discours de la théologie et de la spiritualité ; on pourrait ainsi dire qu'il y a des modalités de réflexivité différente dans les champs de la philosophie dogmatique et de la philosophie spirituelle. La phénoménologie du sujet spirituel est de faire référence à des événements intérieurs qui symbolisent des révolutions intérieures alors que celle du sujet théologique est de faire lien entre des pensées, des concepts, des représentations et Dieu pensé comme concept du concept, ou Idée de la raison pure pour parler comme Kant. L'intention n'est pas du tout la même 
non moins que ses effets de subjectivation.

\section{A/ LOGIQUe de LA ThÉOLOGIE}

14 Il s'agit de penser la cause des causes, le premier moteur du livre lambda de la Métaphysique d'Aristote, le premier moteur immobile, c'est-à-dire, le premier être qui est au fondement de tous les mouvements du monde, auquel nous désirons atteindre, que nous désirons être, qui est sans fondement, et à l'origine de tout mouvement. Dieu, cause sans cause, cause des causes, cause première, cause et fondement, principe de la causalité et de tout le plérôme, et commencement de tout ce qui est, donc de l'être luimême, et donc être autrement qu'être, pour reprendre le titre d'un livre de Lévinas paru en 1974. En fait, Dieu est le tout et le un de la philosophie grecque, Hen kai Pan.

\section{B/ Logique de la Philosophie}

La philosophie est d'emblée un acte de langage et de praxis aléthurgique (Foucault, 2004) qui est recherche interrogative (dialectique, maïeutique, art et technique discursifs) de la vérité à partir des discours et des énoncés autant que des objets des savoirs constitués et des opinions en usage dans la Cité.

La théologie met de façon dogmatique une transcendance comme Un et principe au fondement des choses, du cosmos, du tout : l'exemple des présocratiques dont nous parlerons plus bas est patent pour montrer le caractère à la fois holistique et principiel $\mathrm{du}$ fondement comme cause et fin dans leurs pratiques discursives et comme techniques d'existence (Hadot, 2019).

La philosophie met le logos, l'argumentation, la rationalité au principe de la raison suffisante et du sens des énoncés ; elle parle dans le champ du savoir et du savoir qui se sait lui-même comme savoir vrai, qui s'éprouve à l'aune du réel des arguments, des preuves, des déductions, des raisonnements.

\section{C/ LOGIQUE DU DISCOURS ET DE LA PAROLE}

Parler, dire vrai, faire l'acte de l'acte, et non pas celui du passage à l'acte, c'est s'adresser à quelqu'un pour lui dire, lui parler, lui signifier quelque chose. La philosophie fait le pari du logos, c'est-à-dire, de la parole qui fait de l'énoncé un énoncé performatif, pratique et parrésiastique.

En ce sens, la parole du philosophe, même si elle n'échappe pas au présent de son énonciation (Hegel, 1821 ; Foucault, 1973), est situé dans un univers de sens que Merleau-Ponty appelait du nom de chiasme, situé entre le corps, la chair et l'histoire. Le sens de son discours est toujours situé mais ne se réduit pas à l'empirique de sa situation. Il excède par son intention, son intentionnalité, le présent de sa situation car il est épochal, il vise un moment infini de son parcours et s'adresse à l'universalité, la pluriversalité de l'humanité située dans l'histoire mais l'excédant tout à la fois.

Les concepts et les idées de la philosophie sont ainsi marqués par l'histoire qui les porte mais, l'histoire de la philosophie dépasse le cadre strict d'une chronologie et d'une simple énumération dans le temps passé des idées des penseurs. La logique de l'histoire de la philosophie n'est pas une logique du sens passé des philosophies du passé ; l'éternel présent de la philosophie repose dans l'infinie pratique des interprétations et lectures du passé à l'aune et à l'aide des apories du présent. 
Ainsi, les concepts et les idées des présocratiques sont pré-philosophiques en ce qu'ils sont encore pris dans une physis, dans une philosophie de la nature ou une cosmologie. Il faut l'intention morale au sens humain du terme pour que la philosophie descende du Ciel pour venir sur Terre avec Socrate, et que la sagesse devienne, selon le mot de Pythagore amour de la sagesse, désir et tension vers la sophia, philosophie justement.

Les auteurs comme Anaximandre dont le concept clé est l'apeïron, Anaxagore, avec le nô̂s, Héraclite, avec le feu ou la guerre, Thalès avec celui de l'eau, etc., vise à chercher une Idée directrice non pas régulatrice au sens de Kant mais principielle qui fonde leur savoir sur un principe Un et unique permettant de systématiser le Tout et le Multiple des choses de la nature et de la surnature, du cosmos tout entier afin de le sortir du chaos et de l'indéterminé.

La philosophie commence avec Socrate, qui dit qu'elle vient de l'interrogation sur soi et sur l'humanité ("Connais-toi toi-même!»); le mot philosophie viendrait de Pythagore, signifiant alors l'amour de la sagesse, car il y a abandon de la recherche de l'être, ou de la possession de la vérité, pour la recherche de la vie meilleure pour l'homme. Ainsi, Platon se demande quelle vie est la meilleure des vies possibles et quelle est la plus juste des vies, ou ce qu'on appelle vertu ou beauté, ou courage ou amour ou foi (Platon, République; Ménon; Phèdre; Lachès; Banquet). La question du Bien est corrélée à celle du savoir, et de la science du savoir vrai ou du vrai savoir qui ne se confond pas avec l'ensemble des connaissances et des sciences de l'être ou des domaines ou des régions de l'être.

On ne tuera pas les présocratiques, jamais inquiétés par les pouvoirs, car ils n'agissent pas directement sur la Cité et sur la liberté des hommes ; Socrate est mortel et poursuivi car il est philosophe (il a le souci de soi et de l'autre comme agent et acteur dans la Cité) et non pas parce qu'il pense ou développe des catégories mentales spécifiques, à l'instar du sophiste ou du rhéteur. Il y a une géométrie, une mantique, une herméneutique et une logique ou une cosmologique qui précèdent la philosophie ; Socrate interroge la vérité de la vie des hommes et le pourquoi de leur décision. Il demande les raisons de leur liberté et de leurs actions, de leur praxis spécifique. Il est donc politique car il demande les raisons des actions et des paroles des hommes dans leur façon de persuader l'autre, de penser le monde et d'articuler leur savoir et leur connaissance en vérité de la vie qu'ils mènent et veulent faire mener à la collectivité, à la Cité.

La logique de la philosophie est avant tout celle du logos, de la parole actée, de la parole en acte, parole qui agit et franchit un seuil de vérité, et enlève la doxa au règne imprécis de l'inconstance et du caprice de l'immédiat. Elle est parole, ou discours, qui énonce ce qu'elle désigne, à et pour quelqu'un à qui elle s'adresse, et qu'elle veut persuader de partager une vision du monde, de l'être, de la vie, des valeurs et des vérités.

Elle vise à trouver un accord, un consensus, un universel dans la recherche commune des définitions et des médiations pour arriver à comprendre ou à savoir ce qui fait sens dans le langage en travail sur ce qu'il énonce ou bien annonce. La parole du philosophe n'est pas de l'ordre du signifiant seul car elle va au-delà des mots et de l'ordre du discours, autant qu'elle le peut, dans un style singulier ou le sujet parlant assume le poids et la limite de son énoncé dans la poursuite, la persévérance, la résistance à la fragilité du sens dans le chaos de l'histoire ; elle permet ainsi à la vie philosophique d'être arrachée au non sens et à l'absurdité de la violence pure qui dissolvent la possibilité de donner au néant et à l'injustice une substance que récuse l'historicité singulière du travail du philosophe dans l'ordre du langage qu'il reçoit et 
qui le travaille autant qu'il le travaille dans son œuvre de chaque jour, de tous les instants, d'une vie qu'on peut et doit vouloir, de part en part, marquée par la création continue.

Je vous remercie pour votre attention et reste à votre disposition pour 16 d'éventuelles questions et prolongations de mon étude sur les liens entre philosophie et spiritualité.

\section{BIBLIOGRAPHIE SÉLECTIVE}

ARISTOTE (2014). Euvres complètes. Paris : Flammarion, trad. P. Pellegrin, CORBIN H. (2007). Temple et contemplation. Paris : Entrelacs.

DERRIDA, J. (1967). L'Ecriture et la différence. Paris : Seuil.

FOUCAULT M. : Les mots et les choses (1966) ; L'ordre du discours (1973) ; L'archéologie du savoir (1976). Paris : Gallimard.

FREUD S., Au delà du principe de plaisir (1920). Paris : trad. S. Jankélévitch, Payot, 1927.

HADOT P., La philosophie comme éducation des adultes, éd. Vrin, Paris, 2019

HEGEL G. W. F. (1812, 1816, 1820). La Science de la logique. Doctrine de l'être; Logique de l'essence ; Logique du Concept, trad. P.-J. Labarrière et G. Jarczyk. Paris : AubierFlammarion ; Principes de la philosophie du droit (1821). Paris : trad. R. Derathé et J.-P. Frick., Vrin, 1975.

PLATON (2008). CEuvres complètes. Paris : Flammarion, trad. L. Brisson,

LADREAU G. (1985). Discours spirituel et discours philosophique. Paris : Seuil.

lÉVINAS J. (1974). Autrement qu'être ou au-delà de l'essence. Paris : coll. Biblio, Livre de Poche 